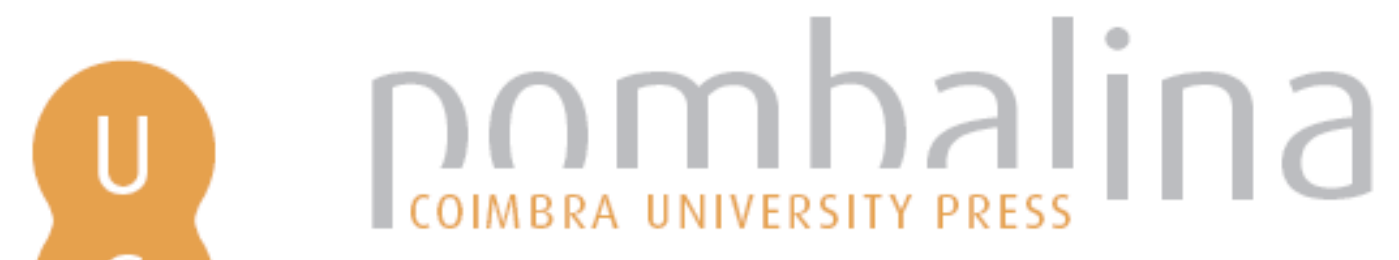

\title{
Société et cultus à l'époque de Martial
}
Autor(es):
Robert, Jean-Noel
Publicado por: Humanísticos; Departamento de Estudos Clássicos, Centro de Estudos Clássicos

\author{
URL \\ persistente: \\ URl:http://hdl.handle.net/10316.2/34630 \\ DOI: \\ DOI:http://dx.doi.org/10.14195/978-989-26-0901-0_4
}

Accessed : $\quad$ 26-Apr-2023 12:01:20

A navegação consulta e descarregamento dos títulos inseridos nas Bibliotecas Digitais UC Digitalis, UC Pombalina e UC Impactum, pressupõem a aceitação plena e sem reservas dos Termos e Condições de Uso destas Bibliotecas Digitais, disponíveis em https://digitalis.uc.pt/pt-pt/termos.

Conforme exposto nos referidos Termos e Condições de Uso, o descarregamento de títulos de acesso restrito requer uma licença válida de autorização devendo o utilizador aceder ao(s) documento(s) a partir de um endereço de IP da instituição detentora da supramencionada licença.

Ao utilizador é apenas permitido o descarregamento para uso pessoal, pelo que o emprego do(s) título(s) descarregado(s) para outro fim, designadamente comercial, carece de autorização do respetivo autor ou editor da obra.

Na medida em que todas as obras da UC Digitalis se encontram protegidas pelo Código do Direito de Autor e Direitos Conexos e demais legislação aplicável, toda a cópia, parcial ou total, deste documento, nos casos em que é legalmente admitida, deverá conter ou fazer-se acompanhar por este aviso.

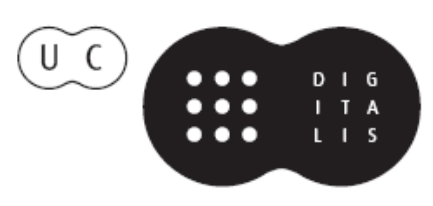




\section{Toto notus in orbe Martialis}

\section{Celebração de Marcial \\ 1900 anos após a sua morte}

Coordenação

Cristina de Sousa Pimentel

Delfim F. Leão

José Luís L. Brandão

Instituto de Estudos Clássicos

Centro de Estudos Clássicos e Humanísticos

UNIVERSIDADE DE COIMBRA
Departamentō de Estudos Clássicos Centro de Estudos Clássicos UNIVERSIDADE DE LISBOA 


\title{
SOCIETE ET CVLTVS A L'EPOQUE DE MARTIAL
}

\author{
JEAN-NOËL ROBERT
}

Faculté Libre de Paris

Résumé: Martial est un poète réaliste qui a «le goût de l'homme» et pratique la franchise du verbe. Il dénonce les dérives de la société et, ravalé au rang de client, il se sent méprisé et se range au nombre des pauvres. Mais qu'est-ce que la pauvreté ? Le seul critère d'appréciation sociale et morale est l'argent. Toutefois, la société n'est pas seulement divisée entre ceux qui en ont et ceux qui en manquent. L'étude rapide des besoins et des revenus fait apparaître qu'il existe une troisième catégorie, celle des exclus qui, à l'instar du Nestor de Martial, sont «les damnés de la terre». Les autres vivent dans deux mondes parallèles et ne se croisent guère qu'en deux moments clés: la salutatio et la cena dont il faut examiner les codes sociaux.

La mentalité romaine a changé depuis la fin de la République; la définition du cultus également, en conséquence. La culture est affaire de fortune. La société s'organise comme un spectacle dans lequel les riches sont au centre de la représentation, ce qui implique pour eux une discipline particulière. Le cultus nécessite une parfaite maîtrise de soi, du corps et du langage (urbanitas). Il est d'abord, à Rome, affaire de sens, de sensualité. Mais de toutes les expressions sensuelles, c'est l'oralité qui définit le mieux la culture romaine. Celle-ci se manifeste à son plus haut niveau lors de deux moments majeurs de la vie du riche: la cena et la recitatio. L'un et l'autre symbolisent la double opération d'ingestion-digestion qui définit la culture et procure un plaisir quasi érotique à l'homme cultivé.

Le peuple n'est pas pour autant banni de cette jouissance orale: la dicacitas est une caractéristique de la mentalité romaine, et un sport national. Le sarcasme est un ludibrium, et l'obscénité une forme de viol verbal dont le but consiste à contraindre l'autre au silence, donc à le dépouiller de son pouvoir de citoyen. 
La parole est l'arme du uir, et sa force toute symbolique. Toutefois cette parole tonitruante masque la vraie pudor, celle des sentiments et des angoisses. Carpe diem, dit aussi Martial, laissant sourdre sa peur de la mort. Mais cette pudor reste du domaine du non-dit, laissant juste affleurer une fragilité trop humaine.

Martial peut être considéré comme le premier poète latin réaliste, dégagé de la gangue des métaphores mythologiques, dont la qualité des vers prouve le talent. Il nous offre une peinture de la société nimbée d'une rageuse authenticité.

Martial est un humaniste en ce sens qu'il s'intéresse à l'homme, et ne néglige aucun des traits de la condition humaine. Son œuvre «a le goût de l'homme» (hominem sapit) ${ }^{1}$, et le manque de profondeur dont on l'accuse est surtout dû au fait qu'il préfère suggérer, croquer plutôt qu'analyser - ce qui n'est pas l'objet de l'épigramme. Son humour féroce, sa causticité font parfois oublier sa franchise, "l'allègre franchise des mots» (lasciuam uerborum ueritatem), comme il l'annonce dans la préface de son premier livre. Pourtant Martial n'est pas «un tueur», il ne s'en prend pas aux personnes ${ }^{2}$. Le ton est celui des Jeux de Flore ou des Saturnales ${ }^{3}$. L'obscénité n'est pas gratuite, elle joue un rôle symbolique. En définitive, le poète pose sur ses contemporains un regard plutôt tendre en même temps qu'ironiquement acerbe. $C^{\prime}$ est la vie qui l'intéresse, dans sa vérité et sa complexité, sans tabou ${ }^{4}$. Il ne résiste pas au plaisir d'un bon mot. Mais le ton se durcit lorsque, de quelques formules assassines, il dénonce les dérives de la société. Plus que le moraliste, c'est alors le peuple qui parle par sa voix.

${ }^{1}$ Martial, Epigrammes 10. 4. 10 [toutes les références à Martial renvoient aux Epigrammes].

2 Id. 10. 33. 10 : Hunc seruare modum nostri nouere libelli / Parcere personis, dicere de uitiis (Mes petits volumes ont appris à garder cette mesure: épargner les personnes, censurer les vices. - trad. H.J. Isaac) Cf. aussi 1. praef.

${ }^{3}$ Id. 1. praef.: Epigrammata illis scribuntur qui solent spectare Florales / Et 11. 15. 11-12: Versus hos tamen esse tu memento / Saturnalicios... (N'oublie pas que tu as sous les yeux des vers de Saturnales...)

${ }^{4}$ Id. 8. 3. 20: adgnoscat mores uita legatque suos. Et 10. 4. 8: hoc lege quod possit dicere uita "Meum est». (Lisez plutôt ce dont le monde des vivants peut dire: «cela m'appartient!») 
L'œuvre de Martial marque un évolution importante de la poésie à Rome en ce qu'elle dénonce une réalité sociale qui attise la haine du poète envers l'injustice sociale dont il s'estime victime. Lucilius traitait de politique, Catulle (qui inspira Martial) se consacra aux affres de sa passion amoureuse, Martial se focalise sur les dérives de la société.

Il se sent misérable, rejeté par les grands qui, jadis, se flattaient d'être entourés de poètes, méprisé et ravalé au même rang que les pauvres auxquels il voue lui-même un mépris appuyé (comme son ami Juvénal). Il n'est que client. Encore faut-il savoir exactement ce que recouvre ce mot à l'époque de Domitien. Il s'estime pauvre. Mais qu'estce que la pauvreté à Rome à son époque?

En fait, Martial ose provoquer ses contemporains en abordant crûment la seule question qui soit au centre des préoccupations de chacun : celle de l'argent. Depuis la fin de la République, l'argent est le seul critère d'appréciation sociale et morale qui permette de définir les différentes catégories de citoyens. La fortune conditionne donc le rang social : d'une part les honesti, les honestiores, qui assument les responsabilités parce qu'ils sont moralement les meilleurs, ils ont l'honestas (l'honorabilité), la dignitas et l'auctoritas (le prestige et l'autorité morale). Le rang social, qui dépend de la seule richesse, induit les qualités morales. En échange, le devoir le plus important de ces honesti réside dans la pratique de la fides; ils doivent aide et protection aux plus humbles. Ils sont les «patrons».

Les autres, les humiliores, les plebeii, apparaissent comme le contraire des honesti, nécessitant une prise en charge, comme l'on fait pour les mineurs. La différence se voit bien dans l'application du code pénal qui, à partir du IIème siècle, différencie les peines selon le rang social du coupable : aux riches, seulement l'exil, aux humiliores les peines infamantes, comme la flagellation ou la peine de mort. On aurait tendance à conclure que les citoyens de la seconde catégorie sont les clients des premiers.

En réalité, les liens sociaux sont beaucoup plus complexes. Il faut ajouter, du point de vue des patrons, les liens d'égalités et les réseaux d'amitiés. Faire partie d'une même famille, par le sang, l'adoption ou un mariage, implique un lien d'égalité parce que ces «égaux» jouent un rôle économique dans le développement de la fortune du patron. Les amis, eux, sont certes des clients, mais des clients à qui le patron est redevable de services, et qu'il flatte (sincèrement ou non) du titre d'amis. Il faut 
d'ailleurs distinguer plusieurs degrés dans ces amitiés qui se traduisent concrètement par la place que les amis se voient attribuer dans la salle à manger du patron lors de ce grand baromètre social que constitue le dîner.

Ceux à qui le patron donne le nom de «client» ne sont alors que les parasites, indifférenciés aux yeux du patron qui se doit de les entretenir en leur distribuant la sportule quotidienne. La première détresse de Martial est morale : il n'est qu'un «client», anonyme et méprisé. Depuis la République, le rapport de patron à client a profondément changé. A cette époque, le devoir réciproque de fides s'exerçait; le patron avait besoin de ses clients, et réciproquement. L'influence du patron se mesurait au nombre et à la qualité de ses clients, et, pour un client, faire partie de la suite d'un patron renommé et puissant (notamment politiquement) était un honneur et garantissait une certaine considération. Le lien qui rapprochait le client de son patron n'était pas seulement économique. Sous l'Empire, au contraire, ce lien moral n'existe plus, car l'empereur est politiquement le seul patron. Ne subsiste donc que le lien économique, auquel s'ajoute l'occasion d'exercer un contrôle des puissants sur les plus faibles pour préserver l'équilibre social et pallier les évidentes carences de l'administration impériale. Mais le riche patron, dont le sort dépend du pouvoir impérial, n'a rien à attendre de ses clients. On pourrait dire qu'à une relation paternaliste, sous la République, a succédé un rapport de vassalité entre le patron et ses clients. Ceux-ci, dépossédés par le nouveau régime de leur rôle de citoyens dans la cité, n'ont plus qu'une préoccupation en tête : trouver de quoi subsister, et notamment de quoi manger. Décrocher une invitation à dîner devient la grande affaire du citoyen, chaque jour renouvelée. On comprend que cette relation purement matérielle ne satisfasse pas le poète. Il pense sans doute à ses confrères des $\mathrm{II}^{\mathrm{e}}$ et $\mathrm{I}^{\mathrm{er}}$ siècle avant notre ère qui vivaient dans l'entourage des puissants et en retiraient gloire et notoriété. Certes, le poète fut toujours à la solde d'un protecteur (Caton ou Scipion l'Africain pour Ennius, Scipion Emilien pour Térence, Memmius pour Catulle et Lucrèce, Mécène pour Horace et Virgile...), mais au moins celui-ci le distinguait-il de la masse des courtisans. Martial se plaint, de surcroît, que les patrons sont devenus avares, et, quand ils veulent bien écouter les œuvres qu'on leur dédie, daignent à peine les récompenser chichement. Or le poète a besoin d'une certaine liberté d'esprit pour faire fructifier son inspiration. "Qu'il y ait des Mécènes, les Virgiles ne manqueront 
pas», remarque Martial ${ }^{5}$. Juvénal portera la même accusation ${ }^{6}$. Voilà pourquoi les poètes de cette fin de siècle se sentent blessés dans leur dignité et estiment que le défaut de vrais protecteurs est la première explication de la décadence littéraire. Voilà pourquoi aussi une certaine liberté d'expression, qui fait le sel de leur œuvre, est la rançon de leur détresse morale.

Quant à leur situation matérielle, celle de Martial par exemple justifie-t-elle qu'il se fasse passer pour pauvre? C'est l'occasion de citer quelques chiffres et de tenter de définir la notion de pauvreté à Rome. Notre poète vit d'abord sur le Quirinal dans un appartement situé au troisième étage d'une insula, avant de déménager dans une petite maison du même quartier. Il demande d'ailleurs à l'empereur l'autorisation de brancher une dérivation de l'Aqua Marcia pour l'alimenter en eau'. S'il ne roule pas carrosse, il possède néanmoins un attelage de mules ${ }^{8}$, ce qui constitue une forme de luxe, et des esclaves, parmi lesquels un cuisinier qu'il fait fouetter à l'occasion'. Ajoutons une petite ferme près de Nomentum, qui ne semble pas lui rapporter beaucoup, mais dont il est fier. Il aime régaler ses amis avec les produits de sa terre $\mathrm{e}^{10}$, ou offrir les fruits de son verger ${ }^{11}$. Il possède même quelques vignes dont il ne déteste pas le vin à la condition qu'il ait vieilli un peu ${ }^{12}$. Il semble donc plutôt à l'aise, et pourtant, il prétend vivre dans la gêne. La raison en est que les mules, les esclaves et la fermette lui ont été donnés, et ne lui rapportent pas beaucoup. Ils lui coûtent même en entretien. Or, à cette époque, on ne peut vivre décemment et indépendant qu'à partir d'un certain revenu de ses biens. A titre d'exemple, un revenu annuel de 1000 HS nécessite une exploitation d'une quinzaine d'hectares. Et encore cela fait-il à peine $3 \mathrm{HS}$ par jour, soit le revenu moyen d'un ouvrier. Le montant minimum journalier de la sportule a été fixé à 6,25 HS, soit plus du double. On estime que le minimum nécessaire à un adulte pour vivre est de 500 à

${ }^{5}$ Id. 8. 55. 5: Sint Maecenates: non deerunt, Flacce, Marones.

${ }^{6}$ Juvénal, Satires 7. 30.

${ }^{7}$ Martial, 9. 18.

${ }^{8}$ Id. 8. 61. 7.

${ }^{9}$ Id. 8. 23.

${ }^{10}$ Id. 10. 48.

${ }^{11}$ Id. 13.42.

12 105; 10. 48. 19; 13. 119. 
700 HS par an, non compris le logement qui, à Rome, est hors de prix (le loyer d'une chambrette insalubre au dernier étage d'une insula peut coûter trois à quatre fois le salaire d'un ouvrier !). Martial n'est peut-être pas à la rue, mais il ne peut se passer de la sportule et doit donc sacrifier au rituel jugé humiliant de la salutatio.

A titre de comparaison, Pline le Jeune possède un patrimoine de 20 millions de HS, principalement hérités de son oncle. (Rappelons que le cens minimum pour appartenir à la classe sénatoriale est $d^{\prime} u n$ million de HS, et de 400000 HS pour un chevalier - Martial fut élevé à la dignité de chevalier par la grâce impériale, mais sans en posséder le premier sesterce). Pline garantit aussi un salaire annuel à ses affranchis de 840 à 1020 HS, et donna à sa nourrice un bien de $100000 \mathrm{HS}^{13}$ dont elle pouvait tirer un revenu annuel de 5000 HS. Il s'agit là du montant généralement reconnu comme la base de la fortune puisque la loi d'Auguste le fixait comme le plafond au-delà duquel un célibataire sans enfant ne pouvait plus hériter. Encore faut-il bien comprendre que, souvent, la plupart de ces fortunes consistait en biens immobiliers dont on ne pouvait disposer immédiatement : domaines, immeubles de rapport. C'est pourquoi le prêt à usure (avec des taux légaux jusqu'à 12\%) était volontiers préféré comme complément de revenus par les riches ${ }^{14}$.

Martial, dans ce contexte de l'argent roi, apparaît donc presque comme un pauvre. Au moins sauvegarde-t-il sa dignité puisqu'il n'est pas obligé de travailler. Déjà, Cicéron notait que «le travail salarié est sordide et indigne d'un homme de bien». Les travaux manuels sont réservés aux esclaves. (Il faut noter que la hiérarchie dans le monde des esclaves reproduit la même complexité que celle des citoyens, du uicarius, l'esclave d'esclave à l'affranchi enrichi). Même quand il gère ses affaires, un riche n'est pas considéré comme accomplissant un travail, à la différence d'un affranchi qui s'occupe des siennes. Tout est dans le regard porté sur la condition de l'homme. Le travail rémunéré est jugé dégradant parce qu'il rabaisse l'âme et pousse au vice. Or beaucoup de petits citoyens qui n'ont pas assez pour vivre doivent chercher à gagner

${ }^{13}$ Pline le Jeune, Epistulae 6. 3; cf. aussi 10. 58 et 10. 110.

${ }^{14}$ Cf. Martial, 4.37 et Pline, Ep., 3. 19. 8: sum quidem prope totus in praediis, aliquid tamen fenero... (Presque tout mon bien, il est vrai, est en domaines; je touche cependant quelques intérêts...). 
un salaire. Ils sont considérés comme pauvres. Et s'ils restent sans emploi (iners), ils sont traités de fainéants, ce qui suppose également une déchéance morale. L'oisiveté n' est noble que pour le riche... La différence $n^{\prime}$ est alors pas si grande entre le petit citoyen pauvre qui travaille et l'esclave. Parfois même la situation du premier n'est pas plus enviable que celle du second, la privation de liberté mise à part. Ainsi dans les campagnes, le petit paysan pauvre qui se retrouve sans terre par un concours de circonstances ou un autre, s'il ne veut pas venir à la ville grossir la cohorte des miséreux, offre-t-il ses services comme saisonnier dans les grands domaines. Il y accomplit les mêmes tâches que les esclaves, voire souvent les travaux les plus durs pour épargner ceux-ci (qui coûtent cher), et il est commandé par l'intendant du domaine, qui est un esclave de confiance du maître. Quant au colonus, s'il est théoriquement libre, il sera progressivement considéré comme attaché à sa terre puisqu'au III siècle le propriétaire qui vendra son bien l'inclura dans la transaction, et il est ainsi l'ancêtre du serf de la glèbe de notre Moyen Age.

A la ville, le petit travailleur gagne, nous l'avons dit, 3 HS par jour en moyenne, quand les terres de Caton rapportaient 600 HS quotidiens à son propriétaire, ce qui était encore bien peu en regard des revenus de Crassus... Souvent, ces pauvres ne peuvent même pas se loger dans une insula, même au dernier étage, dans une de ces pièces aux cloisons si minces qu'il n'était pas conseillé de s'y appuyer si l'on ne voulait pas risquer de se retrouver chez le voisin. Ni eau ni chauffage (sauf un malheureux brasero), mais avec le droit de vider son vase de nuit dans la cuve située sous l'escalier. Et ce pauvre des villes est encore plus misérable que le pauvre des champs, car il se sent déraciné. Dans la mentalité romaine, c'est la terre qui confère la noblesse aux plus riches et assure au moins une certaine vénérabilité aux autres. Exilé à la ville, le pauvre n'a même plus de " tanière " (pour reprendre l'image de Tiberius Gracchus). De la terre, le Romain de la légende puise sa uirtus, comme Curius Dentatus qui pouvait se permettre de refuser l'or corrupteur des Samnites en chassant ces traîtres de sa modeste ferme de Sabine. Voilà pourquoi Ofellus, le petit paysan libre sur son maigre domaine, évoqué par Horace, ne peut être considéré comme un pauvre. Sans doute n'a-t-il rien à manger que quelques légumes avec un peu de porc, sans doute ne peut-il célébrer les jours de fête qu'en tuant un poulet, mais il est chez lui, sur sa terre, où résident ses Pénates. Et c'est en homme libre qu'il fait des 
libations à Cérès pour obtenir ces hauts épis qui dérideront «son front contracté par le souci».

Certes, pour les citadins, il a existé des distributions de blé à bas prix, voire gratuites, mais il faut se souvenir que ces largesses publiques ne concernent pas tous les pauvres, et surtout pas les plus démunis. Nous savons par exemple qu'en -46, 320000 hommes de plus de dix ans en bénéficiaient. Or César, après avoir installé 70000 prolétaires dans des colonies, réduisit à 150000 le nombre des bénéficiaires. 100000 personnes furent donc laissées pour compte. Il est évident qu'il existait plus de 150000 nécessiteux à Rome, et que les cinq boisseaux octroyés par mois ne pouvaient nourrir qu'un célibataire, non une famille. Mais, de toute manière, ces distributions étaient réservées à des privilégiés, et ne constituaient pas, comme le dit Cicéron, un geste de pure générosité. Il faut que le donateur puisse en retirer un bénéfice politique. Comme Pline le Jeune le dira des riches : «pouvoir s'en vanter, c'est le mobile de leur bonnes actions, non leur conséquence». Ceci exclut les miséreux dont il n'y a aucune reconnaissance à attendre. D'ailleurs, les 150000 bénéficiaires de César se vanteront dans leurs épitaphes d'avoir reçu du blé de l'Etat. On ne voit pas sur quel tombeau les miséreux auraient pu inscrire les bienfaits reçus! Même Salluste, popularis et ami de César, conseille au dictateur de reporter les bénéfices de ces distributions sur les vétérans qui ont servi l'Etat plutôt que d'en faire «la récompense de la paresse». Les pauvres ne sont donc pas seulement pauvres, ils sont exclus de la vie politique et économique. Dans une société entièrement conditionnée par la richesse que l'on possède, ils sont «les damnés de la terre». Le Trimalcion de Pétrone aura la formule lapidaire qui résume tout: «si tu as un as, tu vaux un as». Ces pauvres-là sont des sans-abri qui grouillent dans les rues les plus sordides, dépourvus du premier as qui leur permettrait de manger, et il est impossible d'en évaluer le nombre. Mais le danger qu'il font apparemment courir à la société tout entière par l'insécurité qu'ils font régner montre qu'une part non négligeable de la population romaine vit en marge de cette société. Ils errent dans les venelles fangeuses des quartiers chauds de Subure, de l'Argilète ou du Vélabre - selon Pline l'Ancien, il existe à Rome quatre-vingt-dix kilomètres de ruelles insalubres. Ils dorment sous les ponts, sous les portiques, sous les escaliers, dans les caves. D'autres trouvent refuge dans les tombeaux qui bordent les voies au sortir de la ville et qui servent autant de latrines que de lupanars. D'autres enfin se regroupent à 
quelques kilomètres de Rome, sur la voie Appia, près d'Aricie, au cliuus Aricinus - la montagne des sans-abri. Ils y rançonnent les voyageurs qui n'osent plus emprunter la célèbre route vers le sud sans une vaillante escorte. La faim les rend souvent agressifs, car ces déshérités, craints mais ignorés de tous, ne bénéficient d'aucune aide alimentaire. Ils n'entrent même jamais au Cirque, où les places sont très convoitées lors des jeux. Le pain et les jeux, dont les empereurs font une garantie contre la révolte des indigents et des oisifs, ne sont pas pour eux. Voyez Nestor dont Martial brosse le portrait ${ }^{15}$ : il n'a rien, ni toge, ni foyer, ni lit, «fût-il hanté par les punaises», ni cadenas, ni verrou... Que prétend-il? «Prendre une place dans le commun du peuple»? «Prendre le nom et les apparences d'un pauvre»? Mais il n'est rien, pas même un pauvre. "C'est une imposture,... ce n'est pas la pauvreté, Nestor, que de ne rien posséder.» Son domaine, c'est la rue; sa nourriture, le pain que l'on jette aux chiens; son lot, la maladie qui le laissera mort sur le pavé dans l'indifférence générale. La mort est un spectacle quotidien. Les cadavres des miséreux gisent sur le sol des rues, abandonnés aux chiens et aux oiseaux. Un jour, le futur empereur Vespasien voit entrer dans sa salle à manger un chien qui tient dans sa gueule une main humaine. Qui s'en étonne? Personne. Au contraire, l'événement est fêté comme un heureux présage: celui du pouvoir futur du maître de maison ${ }^{16}$. Au reste, les corps des bébés exposés pourrissent sur les tas d'ordures au coin des rues, et les fosses communes de l'Esquilin accueillent aussi bien les cadavres anonymes que les détritus et les excréments. Nous touchons là à l'horreur de l'autre Rome, celle d'une populace ignorée des riches et dont les êtres pullulent comme des cafards sans avoir plus rien d'humain. Rien ne met mieux en évidence le fossé infranchissable qui sépare les extrêmes de la société que la naïve et désarmante expérience de Sénèque. Le philosophe, ému par la souffrance humaine et désireux, en toute bonne foi, de trouver à la pauvreté quelque vertu, décide d'en expérimenter l'existence pendant deux jours. Non point dans la rue (n'exagérons rien), mais dans une petite cellule. Et pour prouver sa bonne résolution, il n'emmène avec lui qu'une seule voiture d'esclaves! «Un matelas est à terre, et je suis sur le matelas. Deux manteaux font office, l'un de drap, l'autre de couverture.»

${ }^{15}$ Martial, 11. 32.

16 Suétone, Vespasien 5. 
Signe de suprême indigence: ses repas sont si simples qu'une seule heure suffit pour les préparer...

Nous le savons, Sénèque est infiniment plus riche que Martial dont la condition de client dépendant fait, d'une certaine façon, un pauvre qui peut avoir faim ${ }^{17}$ et qui est vêtu $d^{\prime} u n$ mauvais manteau ${ }^{18}$, mais notre poète peut passer pour riche aux yeux d'une fraction de la société qui ne compte pas. Présenter de façon rapide la société de cette fin de premier siècle revient à considérer trois catégories: ceux qui détiennent les richesses (les patrons), ceux qui dépendent des riches (les clients), et les exclus qui survivent hors de tout cadre social. Par le fait, ces derniers sont incontrôlables, et font peur : «je hais les pauvres» crie un anonyme qui, de ces mots, a griffé un mur de Pompéi. Entre les deux premières catégories, nous pouvons parler d'une véritable fracture sociale. Le système clientéliste qui, sous la République, avait le mérite de tisser un lien social vertical, tend à laisser la place à un système de ségrégation dans lequel le seul lien pertinent est de nature horizontale, c'est-à-dire économique. Seule la fortune confère à un homme son honorabilité. Le vice d'un pauvre deviendrait une vertu si, par un coup du sort, il devenait soudainement riche. Notre homme, jusque là ignoré, serait alors écouté et sa parole pèserait le poids de ses millions. La fortune devient l'arbitre social.

Mais - et c'est une évidence de le rappeler - l'argent n'est pas une garantie de uirtus, de dignitas, d'auctoritas... Le riche patron dont la situation n'est justifiée que par l'abondance de sa fortune, va devoir marquer sa supériorité par un succédané de la valeur morale qui lui fait bien souvent défaut. Là commence le jeu des apparences et le monde théâtralisé à l'extrême des signes extérieurs de richesse. A commencer par le mépris, la morgue, indispensables pour bien marquer la distance qui le sépare des ses obligés. Cette attitude n'a pour effet que d'attiser la haine des clients humiliés et de créer un univers d'hypocrisie que Martial stigmatise avec insolence. Dès lors, aucune reconnaissance à attendre du pauvre: la seule chose qui l'intéresse chez le riche est son argent. C'est un dû que le client ne manque pas de réclamer, en moquant au besoin la pingrerie de son patron. Les plus cyniques ne cachent pas qu'ils

17 Martial, 7. 27. 10.

${ }^{18}$ Id. 6. 82. 9. 
attendent la mort de celui-ci dans l'espoir de voir leur nom sur son testament.

Deux temps forts de la journée mettent en scène jusque dans le raffinement les instants où se rencontrent les riches et les pauvres, les patrons et les clients: la salutatio et la cena. La salutatio, selon Martial ou Juvénal, est un chef-d'œuvre d'humiliation pour le client qui doit se lever tôt, partir l'hiver dans la nuit, braver les intempéries, marcher dans la boue qui macule les rues, gravir les pentes ardues, faire la queue parmi d'autres clients, saluer l'esclave plein de morgue qui introduit les visiteurs et, parfois lui glisser dans la main une petite pièce pour gagner quelques places, recevoir la maigre sportule d'une main indifférente, sans presque un regard... L'autre moment clé est la cena. Pour deux raisons: d'abord parce qu'une invitation chez un patron est considérée comme un signe de distinction, ensuite parce que, pour certains, sans cette invitation, point d'espoir de dîner, vu leurs maigres revenus. Toutes les compromissions, toutes les bassesses peuvent trouver une excuse dans l'espérance d'être convié à la table d'un riche. Et tant pis si le pauvre est encore une fois humilié, placé loin du lit du maître, peut-être relégué plus bas encore qu'un affranchi à la moins noble origine, mais mieux considéré en raison de ses meilleurs revenus. Tant pis si l'esclave parfumé ne lui sert que des bas morceaux quand les mets les plus raffinés passent sous son nez en direction des lits principaux. Il faut souvent beaucoup de constance et d'opiniâtreté dans la flatterie du riche, jusque dans les latrines, beaucoup d'humilité, voire de servilité assidue auprès du patron, aux thermes par exemple, pour qu'il finisse par délivrer l'invitation magique, souvent plus par lassitude que par bienveillance.

On peut trouver trop sombre cette peinture de la société dont Martial se fait le chantre féroce et cynique. En réalité, cette mutation du tissu social s'explique très bien par l'évolution du régime politique, la confiscation du pouvoir qui réduit le citoyen à l'otium en le privant du negotium, son ancienne justification. Le voilà oisif, passif, attendant qu'on le nourrisse et le distraie (panem et circenses), et pouvant se montrer exigeant dans sa dépendance. Il faut y ajouter la quasi disparition de l'ancienne aristocratie (les vieilles familles se comptent sur les doigts des deux mains), et, avec elle, des valeurs qu'elles véhiculaient ancestralement. Les nouveaux patrons sont peut-être riches, ils ne se sentent plus aucune obligation morale, aucune responsabilité envers 
leurs concitoyens. Cette évolution ne manque pas d'avoir de lourdes répercussions sur la culture et la morale romaines.

Dans le contexte de cette société ploutocratique où l'argent tient lieu de uirtus, on comprend que le seul souci de la majorité des citoyens soit de tout tenter pour s'en procurer. Il est donc inévitable que la culture soit généralement mise en relation avec les richesses et que seuls les riches aient le loisir d'être cultivés. Pour les autres, l'argent lui-même focalise toutes les prétentions et l'intérêt des masses ne revêt qu'un aspect matériel. Pour s'en convaincre, il suffit d'écouter les personnages du roman de Pétrone, lors de la cena Trimalcionis. Bien manger et boire beaucoup sont leurs seules attentes morales et spirituelles, et la générosité de l'amphitryon l'aune à laquelle se juge sa vraie valeur. "Manu plena, uncta mensa», voilà qui définit un ami et un honnête homme ${ }^{19}$. La bonne chère est le signe de la richesse, et donc du bonheur ${ }^{20}$. Et la richesse constitue le summum du bonheur. D'ailleurs Héméros mesure l'«animi beatitudo» de Trimalcion à l'étendue de ses revenus ${ }^{21}$. Ces convives ont l'excuse d'être des affranchis qui ont dû travailler dur pour s'en sortir et savent ce qu'est la misère. Mais combien de petits citoyens tout aussi pauvres n'ont pas une ambition différente. Le cultus Romanorum ne les concerne pas directement, sinon par imitation et nécessité pratique.

A l'époque de Martial, le cultus est une affaire de riche, et se matérialise par un sens aigu du spectacle et de la mise en scène chez ceux qui ont vocation à exercer le rôle de patron. Les pauvres, hormis les exclus, ne sont d'ailleurs pas étrangers à cette mise en scène puis qu'ils en sont les spectateurs, et ne se privent pas de critiquer. Cet aspect de la culture romaine tient à la tradition de vie collective héritée de la République. La vie privée n'existe quasiment pas pour un riche, ou alors elle s'expose aux yeux de tous. La tradition voulait que tout citoyen soit mobilisé au service de la cité, c'est-à-dire qu'il ne soit pas un simple gouverné, mais aussi un acteur, un agent de gouvernement. Plus son rang était élevé, plus sa dignitas exigeait de lui des vertus autant

${ }^{19}$ Pétrone, Satiricon 43. 4 («main pleine et table grasse»).

${ }^{20}$ Id. 38. 10.

${ }^{21}$ Id. 38. 6. 
publiques que privées. Un noble vivait ainsi sous l'œil de la collectivité, avec le devoir de se comporter comme un exemple pour l'ensemble des citoyens. C'est pour cette raison qu'un censeur comme Caton pouvait chasser du Sénat un noble qui avait osé embrasser sa femme devant leur propre fille: il n'avait pas su garder la maîtrise de soi.

La maîtrise de soi, voilà la grande affaire. Le noble se donne en spectacle, jusque chez lui, lorsqu'il reçoit ses clients dans son atrium à l'heure de la salutatio, ou lorsqu'il préside un banquet dans sa salle à manger. Certes, le décor et le costume sont à la hauteur du rôle et concourent à l'auctoritas du personnage, mais celui-ci n'est pas un acteur comme ceux qui montent sur scène. Ceux-ci, légalement et nécessairement des esclaves, sont moralement répréhensibles, non seulement parce qu'ils s'exhibent en jouant de tout leur corps, mais aussi parce qu'ils se prostituent en revêtant une autre personnalité que la leur. Le riche citoyen, lui, doit démontrer la cohérence entre sa fonction et sa personne. Il ne peut donc se comporter naturellement. Il lui faut extérioriser visuellement la classe à laquelle il appartient et, pour cela, façonner son apparence, construire sa silhouette. L'urbanitas et l'élégance exigent un visage rasé de près, éventuellement lissé à la pierre ponce, une coiffure bien taillée et un vêtement bien ajusté sur un corps baigné qui ne dégage aucune odeur désagréable. Le geste est mesuré, la parole réfléchie, les signes de la politesse rigoureusement observés, comme le baiser sur la bouche que s'échangent quotidiennement les membres de la haute société en signe de reconnaissance ${ }^{22}$. Le peuple ne manquera pas de noter les manquements au savoir vivre et de s'en gausser. Il moquera le barbu, qui singe les philosophes et souligne ainsi son désengagement du cultus du citoyen, donc son éloignement des activités de la vie publique collective ${ }^{23}$, ou le chauve qui prouve ainsi sa négligence et son avancée en âge ${ }^{24}$, trahissant l'idéal de l'éternelle jeunesse du citoyen. La

22 Martial, 7. 95. 1-4: Linus, malgré le froid de décembre, distribue ses «baisers glacés» à «Rome tout entière». Cf. aussi 9. 98: la frénésie du basiator devient objet de satire: Effugere non est, Flacce, basiatores. / Instant, morantur, persecuntur, occurrunt / Et hinc et illinc, usquequaque, quacumque... / (Impossible, Flaccus, d'échapper aux embrasseurs. Ils vous pressent, vous retardent, courent après vous, viennent au-devant de vous, de tous côtés, à tout propos, partout.)

${ }^{23}$ Id. 9. 47; cf. 4. 53.

${ }^{24}$ Id. 10. 83; 5. 49. 
manifestation d'un statut social élevé passe donc par une évidente maîtrise du corps et la conformation au cultus exige un douloureux effort sur la nature, comme le rappelle le double sens du verbe colere: le sens physique qui implique une transformation de la nature, et le sens moral qui suppose un lien entre la civilisation et l'éducation.

Le cultus romain est d'abord affaire de sens, de sensualité. Même une opération intellectuelle relève souvent à Rome de sa matérialisation sensuelle. La sagesse, sapientia, est d'abord la qualité de celui qui est capable de goûter en même temps qu'il connaît: le verbe sapere signifie à la fois avoir du goût et avoir de l'intelligence. Le savoir a une saveur, et le goût devient un travail de l'intelligence.

La culture romaine s'exprime dans la sensualité exacerbée du citoyen. Le Romain est un voyeur. Il aime le spectacle, il est sensible aux charmes physiques d'une femme ou d'un jeunes garçon, et les auteurs nous disent combien il est difficile de protéger la vertu d'une jeune personne ${ }^{25}$. Un banquet ne peut se dérouler sans le ballet de jeunes esclaves vêtus à la grecque, et assez dénudés, dont la présence se justifie par le seul plaisir des yeux ${ }^{26}$. Il est sensible aux odeurs, dans une ville où elles sont très variées et prononcées. Celles du corps sont également révélatrices du degré de vertu morale. L'odeur du poulpe, du bouc ou du sanglier passent pour être les plus repoussantes, et démobilisatrices du désir. Un corps nauséabond est un corps dépravé. Un corps séduisant est un corps propre et parfumé ${ }^{27}$. Le Romain apprécie encore le contact physique, et l'on se souvient avec quelle gourmandise Fronton aimait embrasser son élève Marc Aurèle, même si Sénèque, lui, refusait les baisers de Néron, ou comment Ovide conseille de chasser l'invisible poussière sur la poitrine d'une jeune fille sur les gradins du Cirque. Mais le sens le plus développé reste le goût, et son organe majeur, la bouche, qu'il s'agisse de nourriture ou de paroles. Elle constitue l'instrument de

${ }^{25}$ Sénèque, ad Marciam 24. 3. Pline, Ep. 3. 3. 3-4; 4. 13. 4; 7. 24. 3. Quintilien, Institution oratoire 1. 2. 2; 1. 2. 4; 1. 3. 17; 2. 2. 2-4; 2. 2. 14-15.

${ }^{26}$ Martial, 11. 26; 12.91.

27 Ovide, Art d'aimer 1. 503 sqq., décrit cette «beauté sans apprêt» qui sied aux hommes: élégance simple, toge propre et bien disposée, soulier bien adapté au pied, chevelure parfaitement coupée et ordonnée, ongles coupés, absence de poils hirsutes dans le nez, haleine agréable: le cultus exige la stricte maîtrise du corps et de son apparence. 
la sociabilité. L'oralité sous toutes ses formes définit la culture romaine, elle en est la première condition de transmission et de transformation, qu'il s'agisse de nourriture ou de discours. C'est pourquoi les deux moments importants du cultus romain sont le banquet et la recitatio.

Le banquet constitue pour les sens un véritable festival dont le but est de faire participer le convive de l'harmonie universelle symbolisée par la cena. La salle à manger figure symboliquement l'univers dont le patron qui reçoit se trouve, pour le temps du dîner, le maître. Le plafond représente le ciel, les tables la terre, et le sol le monde des morts. La tenue de banquet, dépourvue de toute ceinture, de tout nœud, est prévue pour laisser librement les énergies cosmiques parcourir les corps qui se trouvent ainsi à l'unisson de cet univers. Tous les sens sont flattés: le goût d'abord, avec les raffinements de la cuisine, mais aussi la vue (avec le décor, la mise en scène, et la beauté des jeunes serviteurs, nouveaux ganymèdes), le toucher (quel plaisir de s'essuyer les doigts dans la chevelure savamment bouclée des jeunes éphèbes!), l'odorat (la cuisine épicée, les parfums épandus, les pétales de fleurs qui tombent du plafond...), l'ouïe enfin, avec la musique, les poèmes offerts par le riche à ses hôtes. Le spectacle, qui peut être culturel, complète ainsi la symphonie des sens pour offrir métaphoriquement la double transformation d'éléments naturels et crus (les ingrédients utilisés en cuisine ou les mots des poèmes) en éléments culturels et cuits (les plats préparés ou les œuvres déclamées, tous deux digérés par le convive-spectateur).

La recitatio, offre le même schéma d'ingestion-digestion que le banquet. Mais dans cette opération, c'est l'animus qui joue le rôle du uenter. L'indispensable condition de convivialité est la même, et l'oralité y tient encore la place essentielle. Le lecteur est le cuisinier qui transforme le texte lu, ce que Quintilien assimile à un processus de cuisson ${ }^{28}$. Les oreilles «goûtent» le discours qui doit être assaisonné (conditus) relevé de sel (sal) romain ${ }^{29}$, avoir du goût (sapere). Le souffle du lecteur (spiritus) donne du sens au texte, transforme la matière écrite, et donc crue (crudus) en élément de culture.

Nous savons en effet que la littérature, à Rome passe par l'oralité, aussi bien celle de l'auteur, qui dicte, que celle du lecteur, qui lit à voix haute. La tacita lectio n'est pas appréciée avant le IVe siècle, et l'usage du

${ }^{28}$ Quintilien, Inst. or. 10. 1. 19; 11. 2. 41. Cf. Sénèque, Ep. 11. 84. 5-7.

${ }^{29}$ Cf. Martial, 8. 3. 19. 
uolumen ne la rend pas aisée. Lire est un acte collectif. A Rome, les mots se prononcent avec amour et gourmandise. L'éloquence a toujours été le signe de la grandeur de l'homme, et Cicéron la place au rang des plus hautes vertus ${ }^{30}$. Le latin ne prend tout son sens, que s'il est lu à haute voix, avec les accents. La musique de la langue signifie. Le jeune homme de bonne famille, qui a été instruit et entraîné dans les écoles (où, sous l'Empire, la parole s'est retirée, fuyant le Forum) accomplit une sorte de cérémonie initiatique comme ceux de la République allaient se faire le palais en plaidant quelque cause au Forum pour se montrer dignes de leurs ancêtres. La recitatio est un passage obligé pour entrer dans la bonne société31. L'exercice permet de montrer que l'on est cultivé.

Car la recitatio n'est pas un exercice facile. Elle nécessite d'abord une parfaite maîtrise du corps. C'est une vraie technique (ars). Le lecteur est assis; ses deux mains sont occupées par l'encombrant uolumen. Il doit porter la voix, la moduler et mettre le ton comme le lui a appris le grammaticus, contrôler sa respiration, ménager sa gorge ${ }^{32}$. Son expression doit rester virile, et il ne dispose que de la voix pour faire vivre le texte. Il ne fait pas de geste du bras droit comme l'orateur. Cette prestation demande une préparation physique car le corps doit être dégagé pour permettre le passage du souffle: la marche, une nourriture frugale et l'abstinence sexuelle sont conseillées ${ }^{33}$. Trop de nourriture bloquerait la parole en provoquant un engorgement. La lecture constitue d'ailleurs une excellente hygiène de vie que Celse $^{34}$ recommande de pratiquer quotidiennement.

A lire ce que Perse ${ }^{35}$ écrit des effets de la lecture sur le corps ou du plaisir quasi érotique qui parcourt un corps bien préparé à la lecture, on comprend que le cultus romain associe très étroitement le corps et l'esprit. La parole est action et peut jouer le rôle d'une arme. Elle constitue l'arme du uir cultivé. Rien n'est donc plus déshonorant que d'être poursuivi par la parole d'autrui, voire privé de sa propre parole.

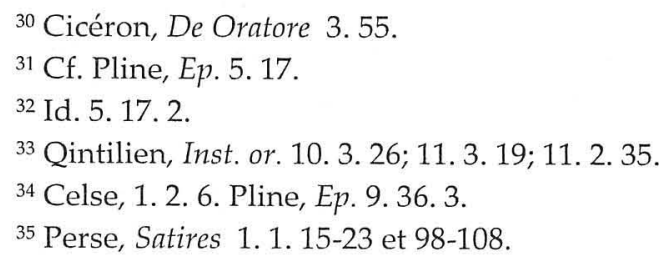


La causticité, la raillerie (dicacitas) est une caractéristique de la mentalité romaine depuis les origines, et l'agression verbale, le jeu des mots qui attaquent publiquement les citoyens sont un sport national propre à toutes les couches de la société. Il traduit particulièrement sous l'Empire la tension qui règne entre les riches et les pauvres, les patrons que l'on envie et les clients qui sont dédaignés. C'est le conflit de l'amicitia et de l'inuidia, qui se traduit par la revendication de la parole comme un pouvoir partagé et par la violence des mots.

Le Romain a toujours eu la langue acérée et le verbe cruel. Depuis le temps des vers fescennins, il se plaît à moquer par des sobriquets, devenus des surnoms, les tares physiques et morales de chacun, même si nul ne lui veut du mal. Pour le plaisir d'un bon mot. Les Caluus (le chauve), Crassus (le gras) ou Claudius (le boiteux) ont su en faire un titre de gloire; d'autres sont plus difficiles à porter, comme Mus (le rat) ou Bibulus (l'ivrogne). Certaines déformations ne laissent aucun doute sur la qualité morale du personnage (Mamurra rebaptisé Mentula par Catulle). Rome est une ville médisante qui met les rieurs de son côté. Dans une société de caractère collectif, chacun juge de tout, et surtout juge les autres. Le peuple est le plus redoutable des tribunaux et il est bien difficile de se relever d'une condamnation au pilori du rire populaire. Même les puissants savent qu'ils ont à se justifier devant l'opinion: Claude est allé devant les prétoriens parler de la conduite de Messaline. Les insultes sont les principales armes qu'utilisent les diverses couches de la société dans cette guerre verbale qu'elles se livrent. Les graffiti fleurissent sur les murs et les bases des statues, y compris celles des empereurs ${ }^{36}$. Des chansons révèlent au peuple les mœurs de leurs dirigeants, comme celle qui courait sur Clodius et Clodia $^{37}$, ou celle que les soldats de César chantaient lors de son triomphe pour rappeler qu'entre lui et le roi de Bithynie, celui des deux qui avait soumis l'autre n'était peut-être pas celui qu'on croit. Les grands eux-mêmes s'envoyaient des libelli, des épigrammes dont Auguste a voulu réduire les effets par une loi ${ }^{38}$. Les auteurs, anonymes, de ces pièces n'hésitaient pas à les afficher ou à les faire chanter dans un lieu public. Tout un quartier pouvait se former en escorte derrière un citoyen coupable de quelque infamie et lui faire un

\footnotetext{
${ }^{36}$ Tacite, Annales 1.72. 4 (sur Tibère). Suétone, Domitien 14 (sur Domitien).

${ }^{37} \mathrm{D}$ 'après Cicéron, ad Quintum 2. 3. 4.

${ }^{38}$ Cf. Digeste, 47. 10: de iniuriis et famosis libellis.
} 
conuicium en l'accablant d'insultes obscènes et de sarcasmes, l'obligeant à fuir sans pouvoir se défendre. Bien plus, l'objet de la vindict populaire était affiché dans un lieu convenu afin que nul n'en ignore: à Rome, sur la colonne Maenius, sur le Forum, près de la Basilique Porcia. Ce genre de proscription était puni par la loi, mais nul n'y pouvait rien et les hommes politiques le redoutaient. Certains gardaient rancune jusque dans la mort, soit en insultant les cortèges funéraires, soit en réglant leurs comptes sur leur épitaphe, accusant tel ou tel au vu et au su des passants censés en lire à haute voix le texte, et devenus par ce fait les propagateurs de la rancœur posthume du défunt.

Rome est devenue une vaste scène de spectacle où se joue le procès des turpitudes. Car le sarcasme est aussi un jeu, un ludibrium, comme lorsque les jeunes gens lançaient des vers fescennins, ou comme dans l'arène, lorsque le condamné, par son supplice, donne au peuple hurlant l'impression d'être la victime de son pouvoir. Il y a, dans cette violence verbale, un vieux souvenir du sacrifice du bouc émissaire. S'y ajoute la valeur apotropaïque de l'insulte obscène, comme en est dotée la statue ridicule de Priape. L'obscénité satirique provoque un rire d'exclusion, et la victime de ce viol verbal en sort déconsidéré, dépouillé de sa dignité d'homme et de citoyen. Les deux insultes les plus violentes que l'on s'adresse en plein Forum sont «irrumo» et «paedico». Toutes deux sont assurément infamante parce qu'elle réduisent celui à qui elles s'adressent à l'état d'objet sexuel passif; ce qui, pour un citoyen, est la plus dégradante des situations. Mais, des deux iniuriae, la première est sans doute la plus avilissante parce qu'elle renvoie à la pure animalité (ruma, la mamelle, s'emploie en général pour désigner le pis d'un animal), et parce qu'elle implique une soumission imposée. L'irrumatio est encore plus vile que la fellatio, parce que cette dernière, bien que moralement répréhensible lorsqu'elle est pratiquée par un citoyen, suppose néanmoins une participation de sa part. Pas l'irrumatio, qui constitue l'humiliation forcée au silence. Elle condamne le citoyen à se taire ${ }^{39}$. La bouche est devenue organe sexuel que l'on peut violer, et qui, comme le culus, départit le citoyen de toute virilité.

Symboliquement, l'obscénité du poète (par exemple de Martial) joue le même rôle que l'irrumatio: elle oblige le lecteur à lire tout haut les mots

${ }^{39}$ Catulle, 74. 5-6. Martial, 3. 96. 3: tel est le sens de la menace: Si te prendero, Gargili, tacebis. (Si je t'attrape, gargilius, je te ferai taire). 
obscènes, à les prendre en bouche, et donc à s'y soumettre en s'avilissant. $S^{\prime}$ il s'y refuse, il est condamné à se taire, il est dépossédé de la parole. Or, dans le cultus romain, l'oralité est l'instrument supérieur de la socialisation, et forcer au mutisme représente la censure suprême qui s'assimile, pour le citoyen, à une dénégation de sa qualité de uir.

L'obscénité, chez Martial comme chez Catulle ou d'autres poètes, n'est donc pas gratuite. Elle a une forte portée symbolique et montre qu'au-delà de l'humour un peu vert ou de la hardiesse verbale dont on la qualifie généralement, elle implique le poète dans une action moralisante dont, il faut le dire, l'esprit de pédagogue du Romain est particulièrement friand. Mais ce manque apparent de pudeur dans le langage (et Catulle comme Martial insistent sur le fait que leurs paroles ne correspondent pas à leurs mœurs ${ }^{40}$ : «ce petit livre et moi n'avons pas les mêmes mœurs.»)n'est qu'un écran tonitruant pour dissimuler la vraie pudor du Romain, celle de ses sentiments et des angoisses qui l'assaillent. La société de cette fin du Ier siècle laisse le citoyen désabusé, et un peu perplexe lorsqu'il s'interroge sur sa place dans le corps social. Cette amertume le fait se retourner sur sa vie, et se poser des questions sur la mort. Certains vers de Martial font écho au célèbre Carpe diem d'Horace: «vivre demain, c'est vivre trop tard: vis aujourd'hui $\diamond^{41}$. Certains n'ont voulu voir dans la légèreté de la formulation que l'expression d'un épicurisme populaire et diffus. A moins que la peur de vieillir ne se dissimule derrière l'apparence d'une indifférence badine. Le poète se moque peut-être $\mathrm{d}^{\prime}$ une femme qui cherche à se rajeunir pour jouir des plaisirs réservés à la jeunesse, mais son cœur s'apitoie devant le spectacle d'une vieille délaissée qui pleure dans une ruelle solitaire. L'inquiétude sourd comme pointe l'angoisse d'Horace devant la menace des neiges qui paralysent de froid le mont Soracte, ou celle des flots bouillonnants de la tempête océane. Le conseil épicurien y prend une tout autre dimension. Le Romain a peur de mourir; même le philosophe. Sénèque ne s'en est pas

${ }^{40}$ Martial, 1. 4. 8: lasciua est nobis pagina, uita proba. (Ma page est libertine, mais ma vie est honnête). Et 11. 15. 13: mores non habet hic meos libellus. (Ce petit livre n'est pas le reflet de mes mœurs).

${ }^{41}$ Martial, 1. 4. 8: lasciua est nobis pagina, uita proba. (Ma page est libertine, mais ma vie est honnête). Et 11. 15. 13: mores non habet hic meos libellus. (Ce petit livre n'est pas le reflet de mes mœurs). 
caché42, et Juvénal a brossé de la déchéance du vieillard un portrait trop exact pour ne pas traduire, du même coup, l'angoisse devant la commune destinée ${ }^{43}$.

Mais cette pudor-là demeurera du domaine du non-dit, occultée par une parole coruscante, seule capable de porter beau en masquant une trop humaine fragilité.

\section{Quelques repères bibliographiques:}

R. DUNCAN - JONES, The Economy of the Roman Empire, Quantitative Studies (Cambridge, 1974).

P. Veyne, Le Pain et le Cirque (Paris, Le Seuil, 1976).

C. VIRLOUVET, Famines et émeutes à Rome, des origines de la République à la mort de Néron.(Rome, Coll. de l'Ec. fr. de Rome, 87, 1985).

A. GIARDINA (ss la dir. de), L'uomo romano (Rome - Bari, 1989).

J.-N. ROBERT, La Vie à la campagne dans l'Antiquité romaine (Paris, Les Belles Lettres, 1985).

J.-N. ROBERT, Eros romain. Sexe et morale dans l'ancienne Rome (Paris, Les Belles Lettres, 1997; éd. poche Hachette Pluriel, 1998).

42 Sénèque, Ep. 12 et 58.

${ }^{43}$ Juvénal, Satires 10. 190 sqq. 